\title{
Desempeño académico en pregrado de la Universidad Nacional Toribio Rodríguez de Mendoza de Amazonas, Perú
}

\author{
Academic performance in undergraduate of the Universidad Nacional Toribio \\ Rodríguez de Mendoza de Amazonas, Peru
}

\author{
César Rafael Balcázar Zumaeta', Segundo Grimaldo Chávez Quintana², Jonathan Alberto Campos Trigoso ${ }^{3}$
}

\section{RESUMEN}

Se tuvo como objetivo evaluar el desempeño de programas académicos de pregrado de la Universidad Nacional Toribio Rodríguez de Mendoza de Amazonas (UNTRM), para lo cual se consideró 21 programas académicos de pregrado con ingresantes en el año 2019 y que presentaban sostenibilidad en la demanda de ingresantes; a través de una investigación descriptiva se evaluó los siguientes indicadores de desempeño: Cantidad de docentes de dictaron cursos, aulas asignadas a los cursos, laboratorios empleados, estudiantes matriculados, y egresados en el 2019; cantidad de publicaciones, cantidad de proyectos asignados. Se determinó que en el año 2019 en los programas académicos en pregrado considerados, 732 profesionales impartieron clases para una población estudiantil de 7121. De otro lado, se identificó que 97 proyectos benefician directamente en términos académicos e investigativos; y además se identificó que hasta mayo del 2020 se registraba un total de 395, donde los autores emplearon la filiación de la Universidad, y de estas 83 publicaciones fueron en revistas de alto impacto. Se concluye que la universidad presenta un progresivo incremento en su desempeño, sobre todo a partir de la nueva ley universitaria y en base a las nuevas políticas de educación superior universitaria para asegurar la calidad educativa en los programas académicos de pregrado.

Palabras clave: Desempeño, calidad, productividad, universidad

\begin{abstract}
The objective was to evaluate the performance of undergraduate academic programs of the Universidad Nacional Toribio Rodríguez de Mendoza de Amazonas (UNTRM), for which 21 undergraduate academic programs were considered with new students in 2019 and that present sustainability in the demand of new students; through a descriptive research, the following performance indicators were evaluated: Number of teachers who taught courses, classrooms assigned to courses, laboratories used, students enrolled, and graduates in 2019; number of publications, number of projects assigned. It was determined that in 2019 in the undergraduate academic programs considered 732 professionals taught classes for a student population of 7121. On the other hand, it was identified that 97 projects directly benefit in academic and research terms; and it was also identified that until May 2020 a total of 395 were registered where the authors used the affiliation of the University, and of these 83 publications were in high impact journals. It is concluded that the university presents a progressive increase in its performance, especially since the new university law and based on the new university higher education policies to ensure educational quality in professional schools
\end{abstract}

Keywords: Performance, quality, productivity, university

\footnotetext{
${ }^{1}$ Maestría en Gestión Pública, Escuela de Posgrado, Universidad Nacional Toribio Rodríguez de Mendoza de Amazonas (UNTRM). Ciudad Universitaria UNTRM, Calle Higos Urco, Chachapoyas, Amazonas, Perú. Correo electrónico: cesar.balcazar@untrm.edu.pe

${ }^{2}$ Docente de la Facultad de Ingeniería Ciencias Agrarias de la UNTRM; Ingeniero Agroindustrial; con el grado de Master en Economía Agroalimentaria y del Medio Ambiente. Correo electrónico: segundo.quintana@untrm.edu.pe

${ }^{3}$ Docente de la Facultad de Ingeniería Zootecnista, Agronegocios y Biotecnología de la UNTRM; Licenciado en Administración de Empresas; con el grado de Master en Economía Agroalimentaria y del Medio Ambiente.Correo electrónico: jonathan.campos@untrm.edu.pe
} 


\section{INTRODUCCIÓN}

Según "Bak (2018) y Pantoja-Aguilar (2019), en la educación pública superior su calidad es proporcional a su inversión, por tanto, fortalecerla garantiza mejorar la calidad teniendo en cuenta la cantidad de recursos limitada y los beneficiarios de la educación reconociendo el papel de la gestión administrativa y gobierno en las instituciones, en el caso de las universidades los procesos de gestión y administración determinan la mejora del quehacer administrativo y el cumplimiento de las metas académicas e investigativas.

Se entiende que la Universidad es un centro de conocimiento, y por el rol y efecto en el progreso de las sociedades y países es importante evaluar el desempeño en términos de productividad y resultados; en la universidad muchas veces inadecuadas gestiones o políticas impiden una mayor competitividad y liderazgo para el desarrollo del país. Por lo que los retos principalmente se encuentran es mejorar la calidad educativa en función de las características sociodemográficas, para lo cual siempre es necesario evaluar los programas académicos con el fin de implementar estrategias que mejoren la acometividad y calidad educativa como institución (Campillo etal., 2019; Coelho etal., 2005)

De otro lado, la gobernabilidad significa la capacidad del sistema universitario en brindar la calidad del servicio para atender las demandas, garantizando el desarrollo integral del estudiante en busca del bien común de la sociedad que es la finalidad pública de toda formación universitaria que se hace visible en términos de productividad y eficacia, para esto el análisis de los indicadores de desempeño es primordial en tomar decisiones a partir de los resultados en la medición de estos indicadores (Dirección General de Educación Superior Universitaria - DIGESU, 2015; Pantoja-Aguilar, 2019).

En el estudio de Villegas y Valderrama (2018) la gestión en una universidad chilena evidenció la estabilidad en el ámbito académico que es producto de la gestión gubernamental asociado al cumplimie-

\section{Tabla 1}

Programas Académicos de pregrado de la UNTRM seleccionados para el estudio.

\begin{tabular}{|c|c|c|c|}
\hline Facultad & Programas académicos & Facultad & Programas académicos \\
\hline \multirow[b]{2}{*}{$\begin{array}{c}\text { Educación y Ciencias de la } \\
\text { Comunicación }\end{array}$} & Educación Primaria & \multirow[b]{2}{*}{ Ciencias Sociales } & Antropología \\
\hline & $\begin{array}{l}\text { Ciencias de la } \\
\text { Comunicación }\end{array}$ & & Arqueología \\
\hline \multirow{4}{*}{ Ciencias de la Salud } & Enfermería & \multirow{4}{*}{$\begin{array}{l}\text { Ciencias Económicas y } \\
\text { Administrativas }\end{array}$} & Adm. de Empresas \\
\hline & Estomatología & & Adm. en Turismo \\
\hline & Medicina Humana & & Contabilidad \\
\hline & Psicología & & Economía \\
\hline \multirow{2}{*}{ Ing. Civil y Ambiental } & Ing. Civil & \multirow{2}{*}{$\begin{array}{c}\text { Ing. Zootecnista, Agronegocios } \\
\text { y Biotecnología }\end{array}$} & Ing. en Agronegocios \\
\hline & Ing. Ambiental & & Ing. Zootecnista \\
\hline
\end{tabular}

nto de metas y una visión ejecutiva de las gestiones adecuados; de otro lado, Quintana etal. (2018) concluye que el desempeño de los programas académicos de la universidad depende de las condiciones institucionales en la formación de los docentes relacionados a composición cualitativa (investigadores, con experiencia, cursando posgrados), la infraestructura y equipamientos disponible, la producción de investigación que contribuyan al contexto educativo, y sistemas de información de planificación y gestión.

Como se ha evidenciado en los antecedentes, el desempeño de las universidades determinadas por los programas académicos es primordial para implementar posteriormente estrategias y medidas que permitan generar una gestión sostenible y productiva de las mismas, que repercutirá a nivel institucional. En el caso de la UNTRM, si bien es una universidad con 20 años de creación ha demostrado estar a la altura de las exigencias educativas que plantea el país, por ejemplo en el 2018 la Universidad recibió más de 4 millones de soles a favor de mejorar la calidad del servicio educativo en el marco de la reforma universitaria, a esto sumado los numerosos proyectos proveniente de fondos concursables accedidos por parte de los docentes para el fomento de investigaciones que beneficia a la institución a través de equipamiento, capacitación e infraestructura adecuada para la formación de profesionales (UNTRM, 2018). Por lo cual, el presente artículo evalúa el desempeño académico de los programas de pregrado de la UNTRM, para así poder tener un panorama del desarrollo como institución y sirva como herramienta para mejorar la calidad de los programas académicos que oferta.

\section{MATERIAL Y MÉTODO}

\section{Programas académicos seleccionados}

De 35 Programas Académicos creados de la UNTRM, se seleccionó 21 programas académicos de pregrado que contaron con ingresantes en el año 2019 y que además evidenciaron sostenibilidad en la demanda académica (Tabla 1) académicas que permiten indicadores de desempeño 


\begin{tabular}{cccc}
\hline $\begin{array}{c}\text { Ing. de Sistemas y Mecánica } \\
\text { Eléctrica }\end{array}$ & Ing. de Sistemas & $\begin{array}{c}\text { Derecho y Ciencias } \\
\text { Políticas }\end{array}$ & $\begin{array}{c}\text { Derecho y Ciencias } \\
\text { Políticas }\end{array}$ \\
\hline \multirow{3}{*}{ Ingeniería y Ciencias Agrarias } & Ing. Agroindustrial & \\
\cline { 2 - 2 } & Ing. Agrónoma & \\
\cline { 2 - 3 } & Ing. Forestal & \\
\hline
\end{tabular}

\section{Recolección de datos}

En la Tabla 2, se detalla las unidades administrativas y base de datos institucionales que fueron fuentes de información para la investigación.

Tabla 2

Fuente según indicador de desempeño estudiado para 21 Programas Académicos de la UNTRM durante el año 2019.

\begin{tabular}{|c|c|}
\hline Indicadores & Fuentes y medios revisados \\
\hline Docentes & $\begin{array}{l}\text { Se contabilizó el número de docentes que dictaron cursos en los programas académicos } \\
\text { seleccionados (2019), la información fue proporcionada por la Dirección de Admisión y } \\
\text { Registros Académicos (DAYRA). }\end{array}$ \\
\hline $\begin{array}{c}\text { Aulas } \\
\text { asignadas }\end{array}$ & $\begin{array}{l}\text { Se contabilizó el número de aulas asignadas por cursos en cada uno de los programas } \\
\text { académicos de pregrado durante el año } 2019 \text {, para ello se recurrió a DAYRA . }\end{array}$ \\
\hline $\begin{array}{l}\text { Laboratorios } \\
\text { empleados }\end{array}$ & $\begin{array}{l}\text { Se realizó una identificación de los laboratorios en la UNTRM mediante la documentación } \\
\text { oficial de la Universidad (UNTRM, 2020) ; luego en base a los cursos impartidos en el } 2019 \text { se } \\
\text { contabilizó los programados según el programa académico y los laboratorios que benefician } \\
\text { directamente a los programas. }\end{array}$ \\
\hline $\begin{array}{l}\text { Estudiantes } \\
\text { matriculados }\end{array}$ & $\begin{array}{l}\text { Se contabilizó el número de estudiant es matriculados en } 1 \text { os semestres } 2019 \text {-I y } 2019 \text {-II de los } \\
21 \text { programas académicos de pregrado seleccionados, información que fue proporcionada por } \\
\text { DAYRA. }\end{array}$ \\
\hline Egresados & $\begin{array}{l}\text { Mediante DAYRA, se recolectó la información del número de egresados en el año } 2019 \text { (I y II } \\
\text { semestre) de los programas académicos seleccionados. }\end{array}$ \\
\hline Publicaciones & $\begin{array}{l}\text { Se recurrió a la información recolectada por la Dirección General de Difusión, Publicación y } \\
\text { Transferencia, luego la información de las publicaciones de la Revistas de la UNTRM, y } \\
\text { posteriormente se obtuvo el reporte de Scopus y el Directorio del CTI (Concytec, 2020). Para la } \\
\text { contabilización se identificó las publicaciones donde los autores emplearon la filiación UNTRM } \\
\text { y la producción científica fue la considerada hasta el mes de mayo del } 2020 \text {. }\end{array}$ \\
\hline $\begin{array}{l}\text { Proyectos } \\
\text { asignados. }\end{array}$ & $\begin{array}{l}\text { Para los proyectos se recurrió al Banco de Inversiones (Ministerio de Economía y Finanzas, } \\
\text { 2020); y los proyectos indicados en resoluciones (UNTRM, 2020). Se identificó el objetivo } \\
\text { general para determinar si beneficia a un programa académico. }\end{array}$ \\
\hline
\end{tabular}

Procesamiento de datos: En base a estos indicadores de desempeño académico, los resultados se contabilizaron para cada programa académico de pregrado, para demostrar el desempeño actual de las variables se empleó estadística descriptiva para apreciar y comparar el funcionamiento de los 21 programas académicos de pregrado. 


\section{RESULTADOS}

\section{Figura 1}

Docentes empleados según programa académico de pregrado en el 2019

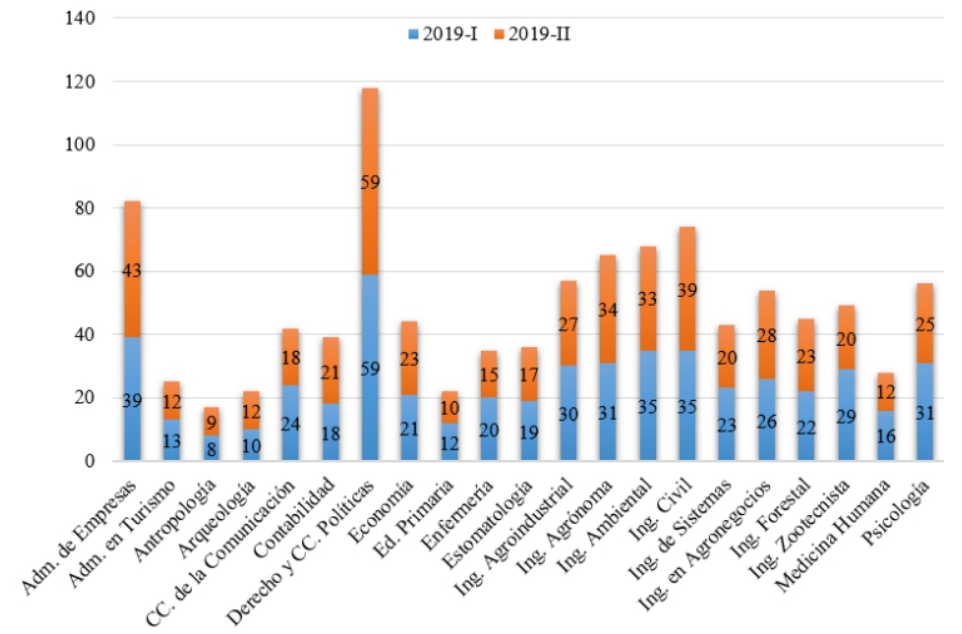

Figura 2

Aulas y Laboratorios empleados en 21 programas académicos de pregrado en el 2019

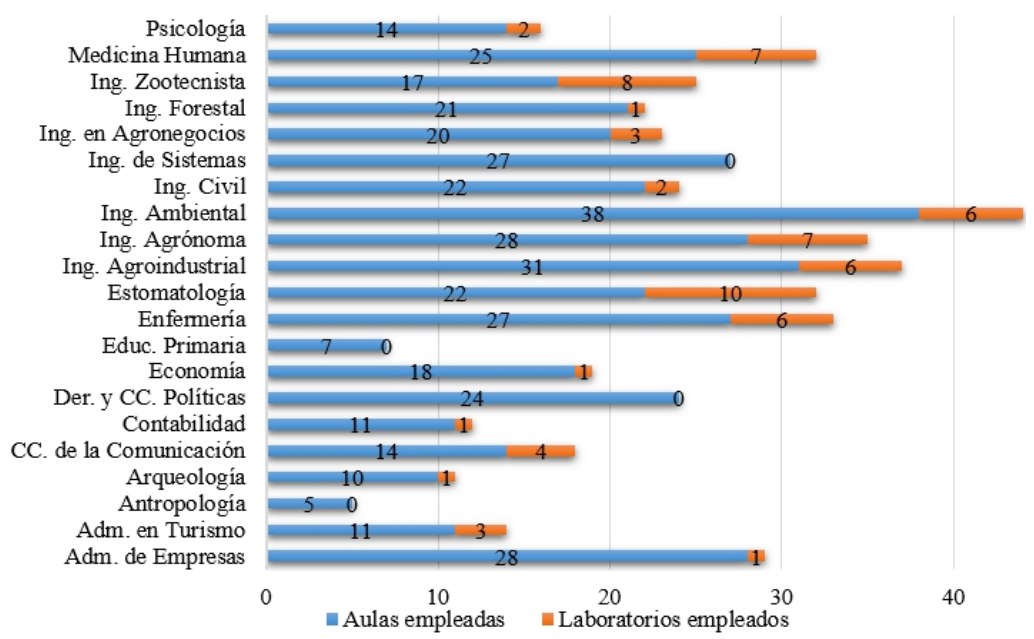

Figura 3

Estudiantes matriculados y egresados según programa académico de pregrado en el 2019

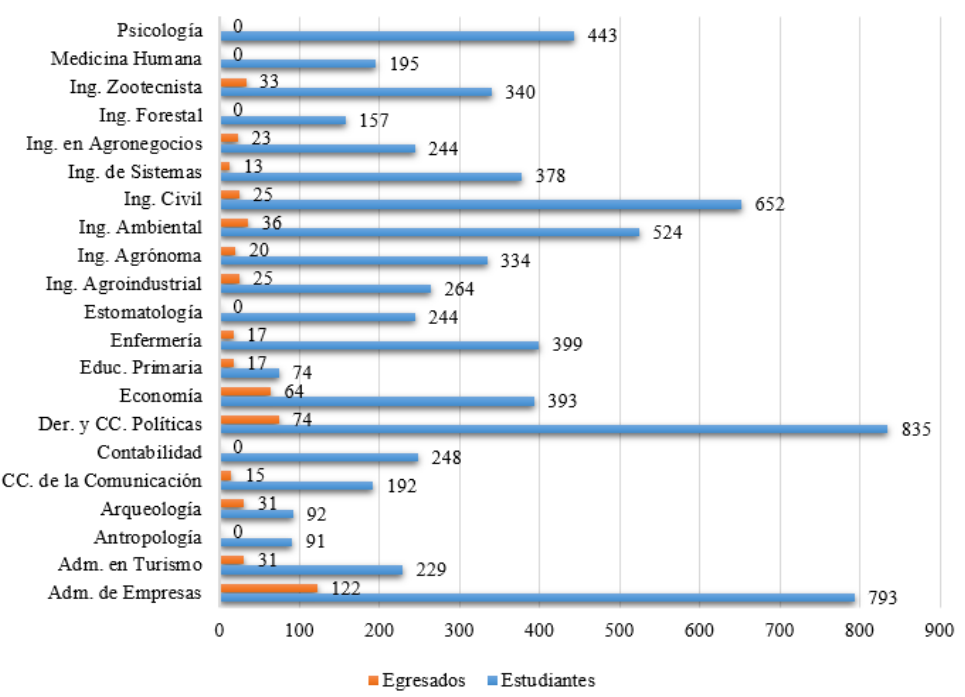




\section{Figura 4}

Proyectos que benefician directamente según fuente de financiamiento de 21 programas académicos de pregrado

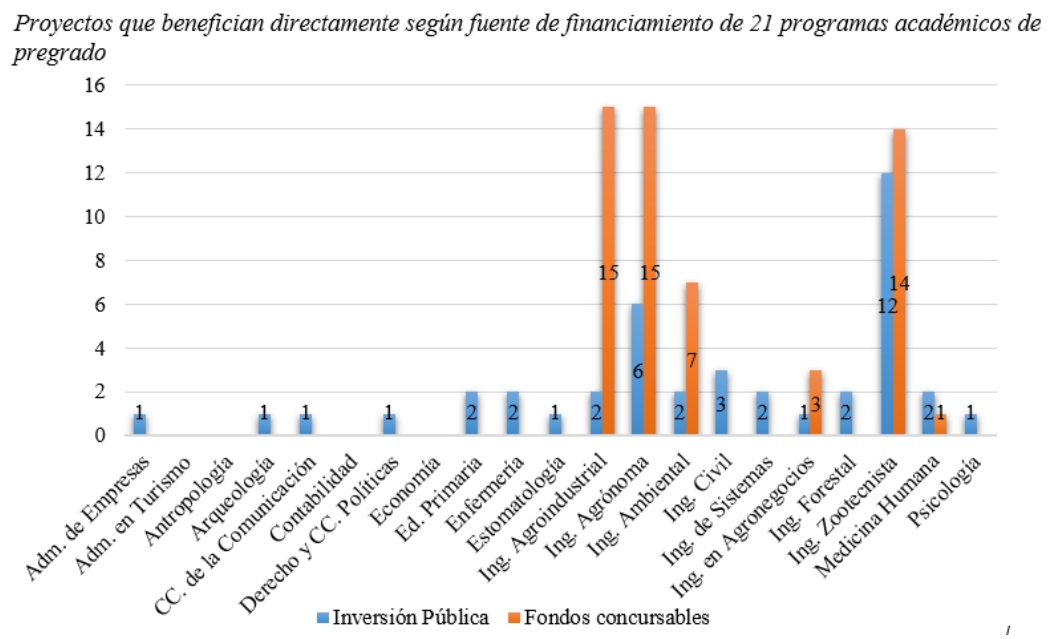

\section{Figura 5}

Proyectos de fondos concursables ganados según años

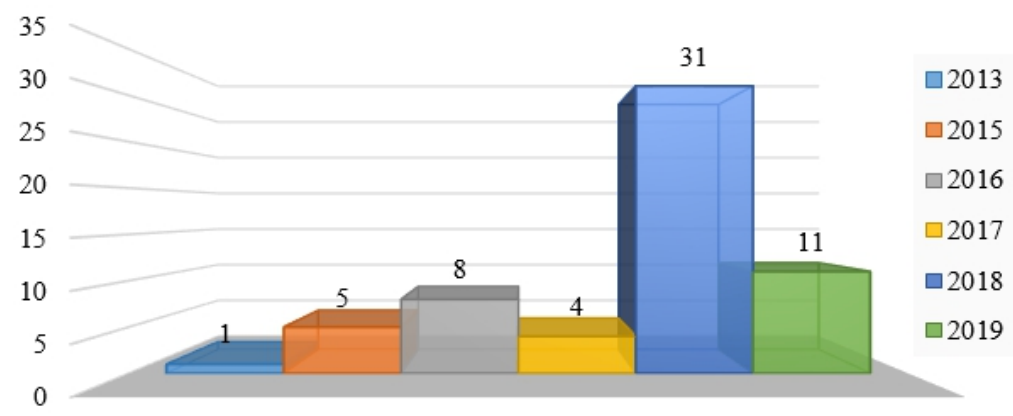

\section{Figura 6}

Producción de publicaciones según fuente de indexación

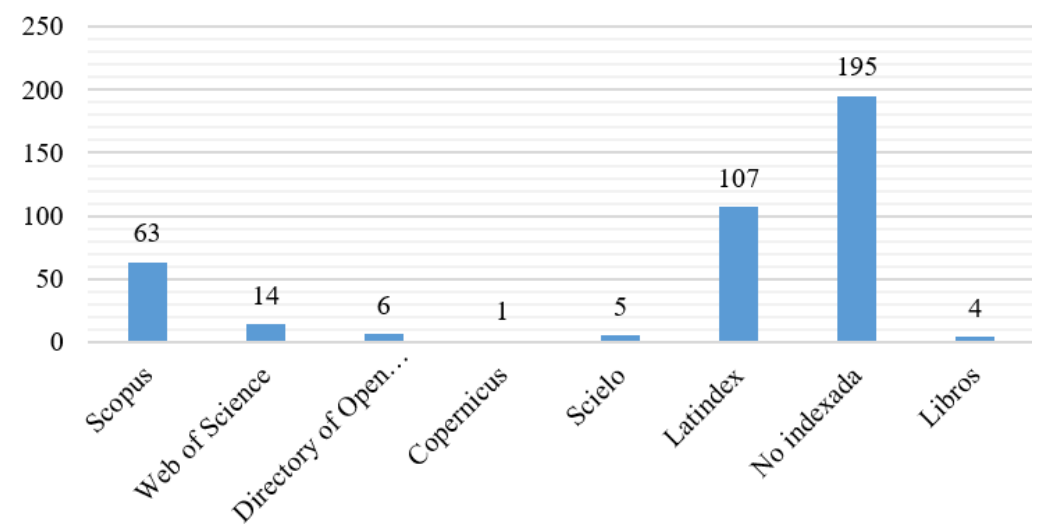




\section{Figura 7}

Cantidad de publicaciones anuales hasta mayo del 2020

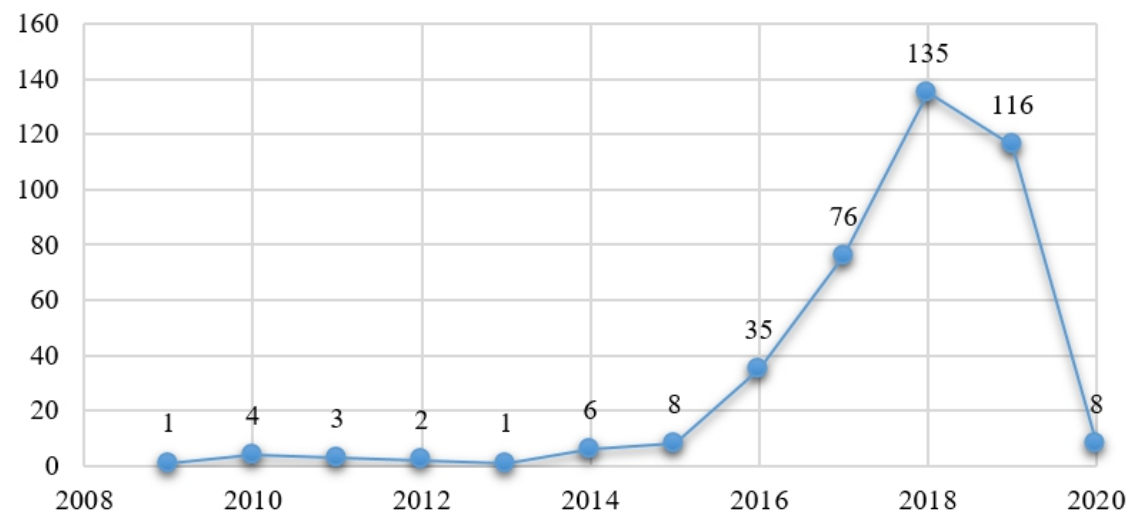

\section{DISCUSIÓN}

En la figura 1, se evidenció que los programas académicos de Derecho y Ciencias Políticas, Administración de Empresas, Ingeniería Civil e Ingeniería Ambiental emplearon un mayor número de docentes para los cursos dictados en el 2019. De acuerdo a esto los programas académicos afines a ciencias económicas representan el 18,6\%, ciencias de la salud el 14,9\%, el área de ciencias sociales y jurídicas el 22,1\%, y el área de ingenierías emplearon $44,4 \%$ del total de docentes; en virtud de los resultados los docentes es un recurso primordial como política de la Universidad y como consecuencia de esto según "Bak (2018) su impacto se evidencia en la formación de alumnos, asimismo la cantidad de docentes empleados es un indicador es de suma importancia pues tiene una relación directa con la producción intelectual que es útil cuando se realizan los rankings universitarios (DIGESU, 2015); además conforme a la última afirmación la producción de los docentes se evidencia en que debe existir un mayor número de docentes investigadores calificados (REGINA) que a la fecha existen un total de 33 investigadores calificados (Concytec, 2021), esta cantidad ha ido aumentando como parte de la política de acreditación de programas de estudios (SINEACE, 2016).

De acuerdo a la figura 2, los programas de Ingeniería Ambiental y Agroindustrial son las que más aulas emplearon a diferencia de Antropología y Educación Primaria para el desarrollo de sus clases; de otro lado, doce programas emplearon más de 20 aulas para sus actividades académicas. En lo referente a la programación de actividades en laboratorios, se observó que 04 programas académicos no emplean laboratorio y que 07 programas emplean para actividades investigativas y académicas más de cinco laboratorios los cuales este último grupo son afines a ciencias reportando que son las que más hacen uso de laboratorios debido a su carácter práctico, por consiguiente esto se relaciona como parte de la política pública delSINEACE (2016) pues se están cumpliendo con las necesidades de equipamiento en función de las competencias en cada programa académico (resaltando ciencias de la salud e ingenierías).

De acuerdo a la Dirección de Admisión y Registros Académicos durante el 2019 a excepción de tres programas académicos todos presentaron un número de estudiantes matriculados entre los dos semestres académicos mayor a 150 (ver figura 3), además que de este último cuatro programas académicos de pregrado presentan una población mayor a los 500 estudiantes siendo el programa de Derecho y Ciencias Políticas con un mayor número de matriculados (835). Además, en la figura 3, se registró un total de 7121 estudiantes matriculados en los 21 programas académicos de pregrado evaluados durante el año 2019; de otro lado, porcentualmente Derecho y Ciencias Políticas $(11,7 \%)$ y Administración de Empresas (11,1\%) contaron con una mayor población estudiantil este resultado es una muestra de la demanda en cuanto a la selección de la carrera a estudiar que está dentro de lo indicado por Trigoso (2019) ya que estas pueden trabajar en diferentes ámbitos. Adicionalmente se deduce que con la implementación de programas académicos de pregrado (inicialmente la Universidad fue creada con cuatro programas) y con la nueva ley universitaria la población estudiantil ha crecido por la calidad educativa pues garantiza el desarrollo profesional, que es una política pública de desempeño como Universidad Pública(DIGESU, 2015).

Asimismo, en la figura 3 se evidenció que en el año 2019 el programa académico de Administración de Empresas y Economía presentaron un mayor número de egresados, de otro lado 05 programas académicos no cuentan con egresados debido a que en algunos casos los programas en pregrado recién han empezado su funcionamiento y no cuentan con la primera promoción de egresados. Como política 
pública, la población de egresados de la Universidad responde a la dirección que necesita el mercado pues es una fuente de profesionales talentosos que están sujeto al empleo disponible que exista y es de suma importancia para los gobiernos en términos de productividad (Campillo etal., 2019; Zhang \& Shi, 2019).

Referente a la figura 4, reportamos que los 21 programas académicos evaluados cuentan con 97 proyectos que benefician a los estamentos de dichos programas, y además se evidenció que cuatro programas no cuentan con proyecto que les beneficia específicamente; de otro lado, de acuerdo al Banco de Inversiones (Ministerio de Economía y Finanzas, 2020) hasta el 2019 existía un total de 75 proyectos declarados viables para la UNTRM de los cuales 42 de acuerdo a su población beneficiaria son destinados a mejorar las condiciones académicas de los programas, además se identificó que cinco programas académicos de pregrado cuentan con proyectos provenientes de fondos concursables. Los proyectos se han constituido como herramientas de beneficio para la población estudiantil en la Universidad, determinado que Ingeniería Agroindustrial (17,5\%), Ingeniería Agrónoma $(21,6 \%)$ e Ingeniería Zootecnista $(26,8 \%)$ son las que cuentan con mayor número de proyectos en contraste a cuatro programas académicos que no cuentan con algún proyecto.

La presencia de proyectos en la Universidad evidencia que el Estado busca financiar acciones para mejorar el servicio educativo (UNTRM, 2018) entendiendo que una alta inversión es proporcional a una mejor educación. De los proyectos que benefician directamente a los programas académicos, de acuerdo a la figura 4 se observa que $43,3 \%$ del total son proyectos de inversión pública (pabellones, laboratorios, etc.) que están bajo el marco del SNIP o Invierte que son recursos ordinarios, y un $56,7 \%$ son de fondos concursables (ver fig. 4). De este último grupo se observa que Ingeniería Agroindustrial, Agrónoma, Zootecnista y Ambiental cuentan con una mayor asignación de fondos concursables lo que las destaca en comparación al resto de programas académicos evaluados, este desempeño involucra un aprovechamiento de los recursos que de por sí ya es limitado para financiar la producción intelectual (publicaciones) y estímulos económicos.

En la figura 5 se observa que en el periodo 2009-2014 durante el primer gobierno universitario post institucionalización solo se alcanzó ganar un proyecto de fondos concursables; además, desde el punto de vista de desarrollo institucional de la Universidad se observa con la implementación de la Ley $\mathrm{N}^{\circ} 30220$ que instó a nuevas elecciones universitarias e implementación de un nuevo sistema de gestión en el periodo 2015-2017 se logró obtener
17 proyectos de fondos concursables, este crecimiento en la opinión de Arias (2017) se debe a la producción de proyectos de investigación por iniciativa de los docentes y profesionales, y actividades administrativas que facilitan el acceso a la inversión de recurso material, humano y financiero. Este indicador de desempeño en términos de investigación se debe a que los fondos invertidos en ciencia y tecnología deben contar un entorno favorable para la investigación.

Hasta mayo del 2020, en cuanto a la producción científica se registraba un total de 395 publicaciones donde los autores emplearon la filiación UNTRM y que pertenecen a uno de los 21 programas académicos que forman parte del estudio, que según Thanassoulis etal. (2018) y Torres-Samuel etal. (2019) para que la producción de publicaciones incremente depende del personal académico como parte de las contribuciones mediante la investigación reflejada en las publicaciones y que son las que posicionan a la Universidades por su capacidad de generar y publicar resultados de investigaciones de calidad. Asimismo, de acuerdo a la figura 6 existía hasta el momento de la recolección de la información un total de 83 publicaciones en revistas de alto impacto en la comunidad científica (Scopus, Web of Science, DOAJ), mientras que existe un total de 195 publicaciones no indexadas y la publicación de 04 libros; en consecuencia, como institución las publicaciones en revistas de alto impacto han ido aumentando progresivamente debido al aporte de la comunidad universitaria. En base a este indicador, podemos evidenciar que el desempeño de los programas académicos en cuanto a las publicaciones contribuyen al sistema universitario, pues como mencionan Torres-Samuel etal. (2019) el Perú es uno de los países mejor posicionados en producción científica; se observa que el desempeño de la universidad tiene una tendencia a publicar en revistas de prestigio a nivel internacional y contar con revistas del mismo tipo con la finalidad de posicionarse como institución.

La figura 7 demuestra que en el periodo 2015-2017 hubo un total de 119 publicaciones, y que en el actual periodo de gobierno estas se han incrementado a la fecha a un total de 259 publicaciones (mayo 2020) donde los autores pertenecen a los programas académicos de pregrado en la condición de docentes, estudiantes y/o profesionales adscritos, observándose que con la Ley Universitaria y el proceso de licenciamiento dichos indicadores de desempeño pasaron por una mejora considerable a diferencia de los años anteriores; estos datos son resultados de un nuevo marco normativo en el sistema universitario donde se tiene como estándar contar con publicaciones realizadas por sus docentes y/o estudiante(SINEACE, 2016); por lo que se infiere que con un modelo de gestión de calidad y 
competitivo hacen posible la visibilidad de las mismas por su capacidad de investigación.

\section{CONCLUSIONES}

Se encontró que los programas de ciencias económicas tienen asignado el $18,6 \%$, ciencias de la salud el $14,9 \%$, el área de ciencias sociales y jurídicas el $22,1 \%$, y el área de ingenierías un 44,4\% del total de docentes ordinarios.

Durante el año 2019, en los programas evaluados se tuvo 7121 estudiantes matriculados en los dos semestres académico, además, de los egresados de ese año, un 3,1\% son del área de ciencias de la salud, un $25,1 \%$ de programas de ciencias sociales y jurídicas, un $32,1 \%$ de ingenierías y $39,7 \%$ de programas afines a ciencias económicas.

Por otro lado, los 21 programas académicos de pregrado evaluados se beneficiaron directamente con 97 proyectos $(43,3 \%$ de inversión pública y $56,7 \%$ de fondos concursables) dotándoles de infraestructura, recurso humano, equipamiento para el desarrollo de actividades académicas e investigativas y bienes de consumo; lo cual se ve reflejado en las publicaciones que hasta el mes de mayo del 2020 suman un total de 395 artículos científicos, donde los autores consignaros como filiación a la UNTRM y pertenecen a uno de los programas académicos estudiados.

En cuanto al desempeño académico de los 21 programas de pregrado que cuentan con ingreso continuo, los indicadores demuestran un progresivo crecimiento a partir de la implementación de un nuevo marco normativo en el 2014, que generó un nuevo sistema de gestión y gobierno universitario. Además, como institución se observa que la política implementada por las autoridades fue efectiva para obtener el licenciamiento, con indicadores que garantizan la calidad educativa con miras a la acreditación.

\section{REFERENCIAS BIBLIOGRÁFICAS}

Arias, F. G. (2017). Efectividad y eficiencia de la investigación tecnológica en la universidad. RECITIUTM, 3(1), 64-83. http://201.249.78. 46/index.php/recitiutm/article/view/92

Bak, H. (2018). Beyond the economy: Education for development. Kasetsart Journal of Social Sciences, S2452315117303260. https://doi. org/10.1016/j.kjss.2018.06.001

Campillo, M., Martínez, A., García, M., Guerrero, L., \& Sánchez, M. (2019). Desempeño académico y egreso en 25 generaciones de estudiantes de la Facultad de Medicina de la UNAM. Educación Médica. https://doi.org/10. 1016/j.edumed.2019.05.003

Coelho, F., Romero, M., \& Yáber, G. (2005). Indicadores de desempeño clave para programas académicos de postgrado. Investigación y Postgrado, 20(2), 123-153. http://ve.scielo.org/scielo.php?script $=$ sci_abs tract\&pid $=$ S131600872005000200005\&lng= es\&nrm $=\mathrm{iso} \& \operatorname{tlng}=\mathrm{es}$

Concytec. (2020). Directorio de Recursos Humanos afines a la CTI [Portal Institucional de CONCYTEC]. Consejo Nacional de Ciencia, Tecnología e Innovación Tecnológica. http://dina.concytec.gob.pe/appDirectorioCT I/index.jsp

Concytec. (2021). Registro de InvestigadoresRENACYT. Directorio de Recursos Humanos afines a la CTI. https://ctivitae.concytec .gob.pe/renacyt-ui/\#/registro/investigadores

Dirección General de Educación Superior Universitaria - DIGESU. (2015). Política de aseguramiento de la calidad de la educación universitaria. Decreto Supremo Nº16-2015MINEDU. Ministerio de Educación Gobierno del Perú. https://www.gob.pe/ institucion/minedu/normas-legales/118310016-2015-minedu

Ministerio de Economía y Finanzas. (2020). Banco de Inversiones. Consulta Avanzada de Inversiones [Portal Institucional del Invierte.pe]. Invierte.pe. https://ofi5.mef.gob. pe/inviertePub/ConsultaPublica/ConsultaA va nzada

Pantoja-Aguilar, M. P. (2019). Indicadores de desempeño académico como predictores de captación de recursos financieros. Convergencia, 26(79). https://doi.org/10. 29101/crcs.v0i79.9222

Quintana, M., Canales, R., Retamal, A., Herrera, C., \& Pérez, C. (2018). Contexto y desafios en Formación de profesores, Universidad de Los Lagos, Chile. Opción: Revista de Ciencias Humanas y Sociales, 86, 450-480. https:// dialnet.unirioja.es/servlet/articulo? codigo $=73$ 38182

Sistema Nacional de Evaluación, Acreditación y Certificación de la Calidad Educativa, SINEACE. (2016). Modelo de Acreditación 
para Programas de Estudios de Educación Superior Universitaria. Sistema Nacional de Evaluación, Acreditación y Certificación de la Calidad Educativa. https://www.sineace.gob .pe/acreditacion/acreditacion-educacionsuperior-universitaria/

Thanassoulis, E., Sotiros, D., Koronakos, G., \& Despotis, D. (2018). Assessing the costeffectiveness of university academic recruitme nt and promotion policies. European Journal of Operational Research, 264(2), 742755.https://doi.org/10.1016/j.ejor.2017.06.04

Torres-Samuel, M., Vásquez, C. L., Cardozo, M. L., Bucci, N., Viloria, A., \& Cabrera, D. (2019). Clustering of Top 50 Latin American Universities in SIR, QS, ARWU, and Webometrics Rankings. Procedia Computer Science, 160, 467-472. https://doi.org/10. $1016 /$ j.procs.2019.11.063

Trigoso, M. (2019). Empresas: Administración de Empresas, la carrera universitaria más demandada. Noticias Gestión Perú. https:// gestion.pe/economia/empresas/administracio n-empresas-carrera-universitaria-demandada -274138-noticia/

UNTRM. (2018). Minedu transfiere más de 4 millones en marco de la Reforma Universitaria-UNTRM. Universidad Nacion al Toribio Rodríguez de Mendoza. https:// www.untrm.edu.pe/es/noticias/1744-minedutransfiere-mas-de-4-millones-en-marco-dela-reforma-universitaria.html

UNTRM. (2020). Resoluciones-UNTRM [Portal Institucional]. Universidad Nacional Toribio Rodríguez de Mendoza. https://www.untrm .edu.pe/es/resolucion.html

Villegas, F. J., \& Valderrama, C. (2018). Lógica de gobierno y de gestión en una universidad estatal de Chile. Opción: Revista de Ciencias Humanas y Sociales, 86, 286-325. https:// dialnet.unirioja.es/servlet/articulo? codigo $=7$ 338178

Zhang, X., \& Shi, W. (2019). Research about the university teaching performance evaluation under the data envelopment method. Cognitive Systems Research, 56, 108-115. 